\title{
The slow-growth high-mortality hypothesis: direct experimental support in a leafmining fly
}

\author{
A K A N E U E S U G I Department of Ecology and Evolutionary Biology, University of Michigan, Ann Arbor, Michigan, \\ U.S.A.
}

\begin{abstract}
Based on the slow-growth high-mortality (SGHM) hypothesis, which predicts that prolonged larval development increases mortality from their natural enemies, studies have often assumed that low quality of plants that slows larval development would function as a defence against insect herbivores. However, empirical support for the SGHM hypothesis has been limited, especially in natural and ecologically relevant contexts.

2. In a leafminer Amauromyza flavifrons Meigen (Agromyzidae, Diptera), the SGHM hypothesis was tested along with four other hypotheses (e.g. prey size, mine appearance, density-dependent parasitism, and plant quality hypotheses) to control for spurious associations between development time and parasitism that are primarily driven by other larval traits. Two host plant species, Saponaria officinalis and Silene latifolia, were grown under varying nitrogen levels, and leafminers developing on these plants were exposed to, or protected from, a natural assembly of parasitoids across the entire course of larval development.

3. On both host plant species, leafminers that survived to an adult stage in the presence of parasitoids had a shorter development time than those in the absence of parasitoids, indicating that parasitoids disproportionately kill leafminers with longer larval development. The results provided concrete evidence for the SGHM hypothesis within the natural ecological context for these interacting species. Moreover, reduced plant quality was associated with higher larval mortality on Sa. officinalis only in the presence of parasitoids, suggesting that low quality could function as indirect plant resistance via SGHM under some tri-trophic interactions.
\end{abstract}

Key words. Larval development time, parasitism, plant quality, plant resistance, tritrophic interactions.

\section{Introduction}

The slow-growth high-mortality (SGHM) hypothesis posits that prolonged larval stages among insect herbivores will extend exposure to, and mortality from, their natural enemies (Moran \& Hamilton, 1980; Clancy \& Price, 1987). This hypothesis has critical implications for the paradoxical evolution of sublethal toxins and low digestibility in plants, which merely retards herbivore growth, thus potentially causing greater tissue loss on plants (Feeny, 1976; Price et al., 1980; but see Wise et al., 2006). Although studies of plant-herbivore interactions often assume the action of SGHM, empirical support for the hypothesis has been equivocal (reviewed in Williams, 1999).

Correspondence: Akane Uesugi, School of Biological Sciences, Monash University, Building 18, Clayton, Victoria 3800, Australia. E-mail: akane.uesugi@monash.edu
Experimental tests of SGHM face three methodological challenges that limit their suitability for testing the hypothesis. First, studies that use different plant species (e.g. Auerbach \& Alberts, 1992; Benrey \& Denno, 1997; Medina et al., 2005) might not detect SGHM if host plant species differ in attractiveness to the enemies of the herbivores. Second, multiple species of natural enemies might attack herbivores at different larval stages, but only a few studies to date have exposed herbivores to their natural community of predators and parasitoids across the entire larval period (e.g. Johnson \& Gould, 1992). Finally, studies that report SGHM often do not control for spurious associations between development time and predation rates that are primarily driven by other larval traits (Hunter, 2003). Even in studies that measure multiple herbivore traits simultaneously, it is often difficult to isolate the effects of individual traits on parasitism if the series of traits are highly correlated with one 
another (Clancy \& Price, 1987; Auerbach \& Alberts, 1992; Cornelissen \& Stiling, 2006).

Here, I test the SGHM hypothesis with a leafmining fly (Amauromyza flavifrons: Agromyzidae; Diptera), using intra-specific variation in plant quality generated by nitrogen addition treatments (Loader \& Damman, 1991; Cornelissen \& Stiling, 2006) within two common host plant species, Saponaria officinalis and Silene latifolia (Caryophyllaceae). Natural enemies are important in mediating plant-herbivore interactions in this leafminer system because larvae are heavily attacked by hymenopteran parasitoids ( 70\%: Scheffer, 1995). To determine whether leafminers with a longer development time are disproportionately attacked by parasitoids, I compared the development time of leafminers that survived to adulthood when they are exposed to and when they are protected from parasitoids (' $\mathrm{P}+$ ' and ' $\mathrm{P}-$ ' treatments, respectively). Because parasitised larvae of A. flavifrons in the test population do not seem to survive to adulthood (i.e. no encapsulation capability, see Materials and methods), this approach essentially measures selection pressure on larval development time imposed by parasitoids. The SGHM hypothesis would predict that the survivors in the $\mathrm{P}+$ treatment would have, on average, a shorter development time than those in the $\mathrm{P}$ - treatment, because individuals with a longer development time are more likely to be killed by parasitoids. By including only the data for survivors in the analyses, I was able to disregard a potential negative effect of endo-parasitoids on leafminer development, as some parasitoids are known to slow their prey growth (Slansky, 1986). The experiment was conducted in the field where leafminers were exposed to a natural assembly of parasitoids during their entire larval development.

To disentangle SGHM from additional causes of differential larval mortality, I simultaneously considered four additional, non-mutually exclusive hypotheses that might explain variation in parasitism: (i) The prey size hypothesis predicts that parasitoids preferentially attack larger prey, presumably because larger host sizes increase parasitoid offspring performance (Clancy \& Price, 1987; Kraaijeveld \& Godfray, 2003; Wang \& Messing, 2004). Under this hypothesis, the final body size of survivors, on average, should be lower in $\mathrm{P}+$ than in the $\mathrm{P}-$ treatments. (ii) The mine appearance hypothesis suggests that the size of leaf mines determines parasitism because parasitoids are better able visually to detect larger mines (Stiling et al., 1999; Cornelissen \& Stiling, 2006). In this case, the mean leaf-mine area of survivors in the $\mathrm{P}+$ treatment is predicted to be smaller than that in the $\mathrm{P}$ - treatment. (iii) The density-dependent parasitism hypothesis predicts that the risk of detection could either increase with neighbour density owing to resource concentration effects (Walker etal., 2008; Singer etal., 2012), or decrease with density as a result of dilution effects (Otway et al., 2005). Thus, the mean neighbour density associated with the surviving leafminers would diverge between the $\mathrm{P}-$ and $\mathrm{P}+$ treatments. (iv) The plant-quality hypothesis suggests that parasitoids are attracted to plants with a higher nutritional quality (here defined as leaf chemical and morphological traits, see Materials and methods) because the herbivores feeding on such plants are likely to be also of high quality (Loader \& Damman, 1991; Hunter \& Price, 1992; Walker et al., 2008). According to the hypothesis, leafminers that survive parasitism should disproportionately come from low-quality plants. Thus, the mean plant quality associated with survivors is predicted to be lower in the $\mathrm{P}+$ than in the $\mathrm{P}-$ treatment. Because the five focal traits (development time, final body size, mine size, neighbour density, and plant nutritional quality) are likely to be correlated, I tested each hypothesis by controlling for variation in the other four traits.

\section{Materials and methods}

Study system

Amauromyza flavifrons is a leafmining fly that feeds on plants in the family Caryophyllaceae (Spencer, 1990). In southeastern Michigan, U.S.A., it is most commonly found on two Caryophyl hosts, Saponaria officinalis and Silene latifolia. Both the leafminer and its host plants were introduced to North America from Europe, re-forming the pre-existing coevolutionary relationship in a new range, but in the absence of other specialist herbivores found in Europe (Wolfe, 2002) that could complicate the field estimates of leafminer life-history traits.

The leafminer is multivoltine, having three to four generations per year. Adults emerge in early June and females lay eggs under the epidermis layer of the host plant leaf. Eggs hatch within 3-4 days and larvae mine for approximately 2 weeks until they exit leaves to pupate in the soil. During its larval development, the leafminer can be heavily attacked by multiple species of parasitoids in the families Braconidae, Pteromalidae, and Eulophidae (Scheffer, 1995). The diet breadth of these parasitoids and their origins are currently unknown. Larvae in early instars are subject to mortality from host feeding by adult female parasitoids (Jervis \& Kidd, 1986). Late instars may be heavily parasitised, with parasitism rates reaching as high as $70 \%$ (Scheffer, 1995). Predation, which was evidenced by ripped mines with no larva, was rarely observed (A. Uesugi, pers. obs.). Despite the high parasitism levels, larvae seem to lack direct defensive mechanisms, such as encapsulation of parasitoid eggs or larvae. For instance, an attack by a Braconid endoparasitoid did not induce melanisation in the leafminers $(n>30)$ from the same source population used in this study (A. Uesugi, pers. obs.). Its restricted movement during the larval period facilitates accurate measurement of each leafminer's life history traits and survival, and a high risk of parasitism makes this system ideal for testing the SGHM hypothesis.

\section{Field experiment}

A field experiment was conducted in July 2007 at Mathaei Botanical Gardens (MBG) in Dixiboro, Michigan, U.S.A. Two species of host plants, Sa. officinalis and Si. latifolia, were grown in pots in the greenhouse under weekly nitrogen treatments until the plants were taken out to the field: a 'low-nitrogen' treatment received $5 \mathrm{ml}$ of $9 \mathrm{mM}$ ammonium nitrate solution $(0.025 \%$ nitrogen), and a 'high-nitrogen' treatment received $5 \mathrm{ml}$ of $1.17 \mathrm{M}$ solution (3.3\% nitrogen). The high-nitrogen treatment mimics the nitrogen levels experienced in fertilised agricultural fields where Sa. officinalis and Si. latifolia often grow. Here, 
nitrogen treatments were used to test the plant-quality hypothesis, as well as to artificially create variation in leafminer's life history traits, as leaf nitrogen levels are known to influence larval development time, body size, and mine size in other leafmining insects (Stiling et al., 1999). Nitrogen treatments also generated variation in neighbour density by changing plant size and leaf availability (see Results). All Sa. officinalis plants were grown from rhizome cuttings collected from one natural patch (probably representing a single clone) and Si. latifolia plants were grown from seeds collected from a single plant. Thus, plant trait variation within a species observed here are predominantly as a result of variation in soil nitrogen level rather than plant genotypic variation. Plants had been growing for 2 months prior to the experiment and some Si. latifolia plants started flowering during the experiment.

Females of the leafmining fly were collected from a $S a$. officinalis patch at MBG in the previous generation. Six potted plants of the same species and nitrogen treatment were placed in a plastic cage where eight 3- to 4-day-old mated females were released for oviposition for $6 \mathrm{~h}$. To maximise oviposition, new females fed with $30 \%$ honey water were used for each oviposition trial. Plants containing the leafminer eggs were kept in a greenhouse under mesh cover for 3 days before they were exposed in the field. This was done so that larger experimental larvae could be distinguished from non-experimental larvae subsequently oviposited by the wild population of the leafminer (Scheffer, 1995).

To avoid competition among larvae, a single larva per leaf was allowed to develop, and the rest were killed by a needle. The number of initial experimental larvae was counted. Half of the plants were bagged with fine mesh to exclude parasitoids $(\mathrm{P}-$ treatment $)$ and the other half were not $(\mathrm{P}+$ treatment $)$. A total of 83 Sa. officinalis (16 High N/P-, 17 Low N/P-, 24 High $\mathrm{N} / \mathrm{P}+$, and 26 Low $\mathrm{N} / \mathrm{P}+)$ and 69 Si. latifolia (13 High N/P-, 17 Low N/P-, 17 High N/P+, and 22 Low N/P+) potted plants were placed in an open field under shade cloth at MBG in a complete randomised design. The number of plants under the $\mathrm{P}-$ and $\mathrm{P}+$ treatments is unbalanced to account for a potential sample loss due to $100 \%$ mortality of leafminers in $\mathrm{P}+$ treatment. The shade cloth shielded plants from direct sunlight and created relatively homogeneous environments between the $\mathrm{P}-$ and $\mathrm{P}+$ treatments, with ambient temperature increased in the $\mathrm{P}-$ treatment only slightly (mean increase $=0.3^{\circ} \mathrm{C}, t=2.2$, $P=0.35)$.

All leaves were checked daily for leafminer development. Each leaf containing a large pre-pupal larva was individually collected in a Petri dish before it emerged from the leaf to pupate in the soil. Most larvae collected in this way pupated in their Petri dish within a day of collection, but ones that did not ( $<3 \%$ of total larvae collected) were excluded from the analysis because they were not exposed to the field conditions during their entire larval period. Development time was calculated as the number of days between oviposition and pupation. Leaves containing leafminer larvae were brought to the laboratory and photographed immediately to estimate mined area at the time of leaf collection using Adobe Photoshop software. Insects were reared to adults at room temperature $\left(25^{\circ} \mathrm{C}\right)$, and the body size of eclosed adult flies was estimated by measurements of thorax length (ImagePro program, Media Cybernetics Inc., Warrendale, Pennsylvania) using the margin of the pronotum and scutelum as landmarks.

\section{Leaf quality measurements}

Leaf quality measures, including the specific leaf area (SLA), water content, $\mathrm{C}: \mathrm{N}$ ratio, and saponin concentration, were estimated for each plant at the end of the field experiment after all leafminers were harvested as above. Five fully expanded leaves were collected from each plant; leaf areas were measured using the ImagePro program, and leaves were weighed before and after they were dried in a $50{ }^{\circ} \mathrm{C}$ oven. The rest of the leaves were dried and weighed to estimate the total leaf area of each plant. The leaf $\mathrm{C}: \mathrm{N}$ ratio of the dried leaf materials was estimated using a Perkins Elmer CNO analyser. The methods used here only measure an average leaf quality of the plant rather than the quality of each leaf that an individual leafminer fed upon. However, because most larvae were found on leaves of similar ages (i.e. mature leaves with no sign of senescence), I assumed that larvae on the same plant experienced similar leaf quality.

Plants in the family Caryophyllaceae are known to contain saponins (Jia etal., 2002) that are potential defensive compounds against generalist herbivores (Goławska et al., 2008), although their effect on specialists, such as A. flavifrons, is not well understood. Because the majority of saponins in Caryophyl plants have quillaic acid as an aglycone (Jia et al., 1998) the concentration of quillaic acid saponins was measured here. Powdered leaf samples $(200 \mathrm{mg}$ ) were extracted with $\mathrm{MeOH}$ and hydrolysed with $2 \mathrm{ml}$ of $1 \mathrm{~N} \mathrm{HCl}$ at $80^{\circ} \mathrm{C}$ for $8 \mathrm{~h}$ to yield quillaic acid. The solution was neutralised by adding $1 \mathrm{M} \mathrm{KOH}$, and extracted with $5 \mathrm{ml}$ of EtOAc three times. Dried compounds were re-suspended in $\mathrm{MeOH}$ containing the cardenolide digitoxin as an internal standard. Quillaic acid concentrations were estimated by reverse-phase high-performance liquid chromatography (HPLC) on a Waters Acquity UPLC with mass spectrometer (Waters Corporation, Milford, Massachusetts), according to the methods in de Roode etal. (2008). Saponin extract from Quillaja saponaria (Sigma-Aldrich, St. Louis, Missouri) was used to generate standard curves relating the concentration of saponins and quillaic acid. Five known concentrations of saponin extract (ranging from 1 to $8 \mathrm{mg} \mathrm{ml}^{-1}$ of $\mathrm{HCl}$ ) were hydrolysed and analysed with HPLC as described above. Using the standard curves, I estimated the amount of quillaic acid saponins in the leaf samples per gramme of dry weight.

\section{Statistical analysis}

All statistical analyses were conducted with $\mathrm{R}$ ( $\mathrm{R}$ version 2.14.1; R Foundation for Statistical Computing, Vienna, Austria). To extract a single measure of leaf morphological and chemical traits that are likely to be associated with the nutritional quality of the plant, I conducted a principal component analysis (PCA) using SLA, water content, $\mathrm{C}: \mathrm{N}$ ratio, and saponin concentrations as variables. PCA analyses were conducted separately for the two host plant species, and the effect of nitrogen and exposure treatments on the first principal component was tested using ANOVA. 
Leafminer mortality in the $\mathrm{P}$ - treatment indicates mortality caused by plant resistance, whereas that in $\mathrm{P}+$ represents mortality jointly caused by plant resistance and parasitism. Leafminer mortality was compared between host plant species and exposure treatment using a generalised linear model with binomial function ( $\mathrm{glm}$ function in R). The interaction between these factors indicates that the parasitism rate differs between host plant species.

To test which of the focal traits are associated with parasitism, I compared trait means of leafminers that survived to adulthood in the $\mathrm{P}-$ and $\mathrm{P}+$ treatments, separately for $\mathrm{Sa}$. officinalis and Si. latifolia. A significant shift in the trait mean between the exposure treatments would indicate that parasitoids disproportionately attack leafminers with respect to that trait. Because the focal traits are likely to be correlated with each other, when analysing each trait, I included the other four traits as covariates. Including plant quality as a covariate had an additional purpose of controlling for observed differences in plant quality between the $\mathrm{P}-$ and $\mathrm{P}+$ environment owing to bagging treatment (see Results). In case of development time, body size and leaf-mine area, I used a linear mixed model (lmer function in R) to include plant as a random effect, as larvae growing on the same plant are likely to express similar phenotypes. For neighbour density and plant quality, the random effect was not included because each larva reared on the same plant had the same density and quality values, and the effect of exposure treatment was assessed using an ANCOVA.

The effects of plant quality on direct and indirect plant resistance were examined in terms of leaf tissue consumption and larval mortality. The amount of leaf tissue consumed (mg) was estimated by first calculating the average leaf-mine area for each plant, and dividing them with SLA estimated for each plant. In each host plant species, I conducted a linear regression analysis with exposure treatment, plant quality, and their interaction as factors. The relationship between per-plant larval mortality and plant quality with and without parasitism was examined using a generalised linear model with binomial function. An interaction between plant quality and exposure treatment would indicate that plant quality differentially affects these resistance measures in the two environments, and may suggest the presence of indirect resistance via enhanced mortality from parasitoids.

\section{Results}

\section{Effect of nitrogen and exposure treatments on plant quality}

Nitrogen treatments successfully created variation in plant quality measure, which was defined as the first principal component (PC1) in the PCA, and explained $62.8 \%$ and $62.1 \%$ of the total variance in Sa. officinalis and Si. latifolia, respectively. On both host species, PC1 was correlated with decreased C: N ratios (species scores: -1.87 and -2.0 for Sa. officinalis and Si. latifolia, respectively) and water content $(-1.68$ and -1.85$)$, and increased SLA (1.73 and 1.76). In Sa. officinalis, the quillaic acid saponin concentration was negatively correlated with PC1 (-1.28), but the saponins were not detected in Si. latifolia. High nitrogen treatment increased PC1 in both plants compared with low nitrogen treatment (ANOVA: $F_{1,74}=127.1$,
$P<0.0001$ for Sa. officinalis, and $F_{1,65}=64.4, P<0.0001$ for Si. latifolia). Saponaria officinalis plants in the bagged treatment $(\mathrm{P}-)$ had higher $\mathrm{PC} 1$ than that in the $\mathrm{P}+$ treatment $\left(F_{1,74}=12.3\right.$, $P=0.0007)$, but the bagging did not affect PC1 in Si. latifolia plants $\left(F_{1,65}=0.095, P=0.76\right)$. No interactions between nitrogen and exposure treatments were found in either host species $(F<1.6, P>0.21)$.

\section{Leafminer mortality to parasitoids}

One thousand four hundred and fifty-two total experimental larvae initiated mines (430 in the $\mathrm{P}-$ and 436 in the $\mathrm{P}+$ treatments on Sa. officinalis and 239 in the $\mathrm{P}-$ and 347 in the $\mathrm{P}+$ treatments on Si. latifolia). Mesh bags successfully excluded parasitoids, with no parasitoids emerging from plants under the $\mathrm{P}$ - treatment. Larval mortality in the $\mathrm{P}-$ treatment reflects mortality caused by plant resistance, and it averaged $31.1 \%$ and $28.5 \%$ for Sa. officinalis and Si. latifolia, respectively. Mortality in the $\mathrm{P}+$ treatment, which is jointly caused by plant resistance and parasitism, amounted for $89.5 \%$ on Sa. officinalis and $68.8 \%$ on Si. latifolia. A generalised linear model reveals a significant host species effect $\left(\chi^{2}=72.45\right.$, d.f. $\left.=1, P<0.0001\right)$, a parasitism effect $\left(\chi^{2}=503.6\right.$, d.f. $\left.=1, P<0.0001\right)$, and an interaction between host species and parasitism $\left(\chi^{2}=10.9\right.$, d.f. $=1$, $P=0.0009$ ), suggesting greater mortality from parasitoids on Sa. officinalis than on Si. latifolia (mean estimated parasitism: $59.6 \%$ \& $47.0 \%$, respectively).

\section{Tests of SGHM and other hypotheses}

On Sa. officinalis, development time ranged from 13 to 16 days. Leafminers that survived in the $\mathrm{P}+$ treatment, on average, had 0.40 days shorter development time than those survived in the $\mathrm{P}$ - treatment when controlled for other variables (LMM exposure treatment effect: $F_{1,74.3}=5.85, P=0.02$, Fig. 1a, Table S1), suggesting that leafminers with a shorter development time were more likely to escape parasitism and survive in the $\mathrm{P}+$ environment. Development time was also negatively correlated with leaf-mine area (coefficient $=-0.11$, $F_{1,329.8}=5.64, P=0.02$ ) and plant quality (coefficient $=0.25$, $F_{1,38.1}=6.11, P=0.02$ ). On Si. latifolia, larval development time ranged from 13 to 17 days, and the survivors in $\mathrm{P}+$ tended to have a shorter development time than those in the $\mathrm{P}-$ treatment (difference $=0.34$ days, exposure effect: $F_{1,60.1}=3.66, P=0.06$, Fig. 1a). Other factors, including plant quality, were not strongly correlated with development time on Si. latifolia (Table S1).

Adult body size did not differ between exposure treatments on either host plant $\left(F_{1,69.4}=0.15, P=0.7\right.$ for Sa. officinalis and $F_{1,47.2}=1.63, P=0.21$ for Si. latifolia, Fig. $1 \mathrm{~b}$ ), nor did the mine size $\left(F_{1,69.4}=0.21, P=0.65\right.$ for Sa. officinalis and $F_{1,46.7}=2.43$, $P=0.13$ for Si. latifolia, Fig. 1c). The mean neighbour density that survivors experienced also did not differ between exposure treatments on either host plants $\left(F_{1,354}=1.9, P=0.17\right.$ for $S a$. officinalis and $F_{1,239}=0.37, P=0.54$ for Si. latifolia, Fig. 1d). Finally, the mean plant quality associated with surviving larvae did not differ between exposure treatments on Sa. officinalis $\left(F_{1,354}=0.81, P=0.37\right)$, but that on Si. latifolia was lower in 


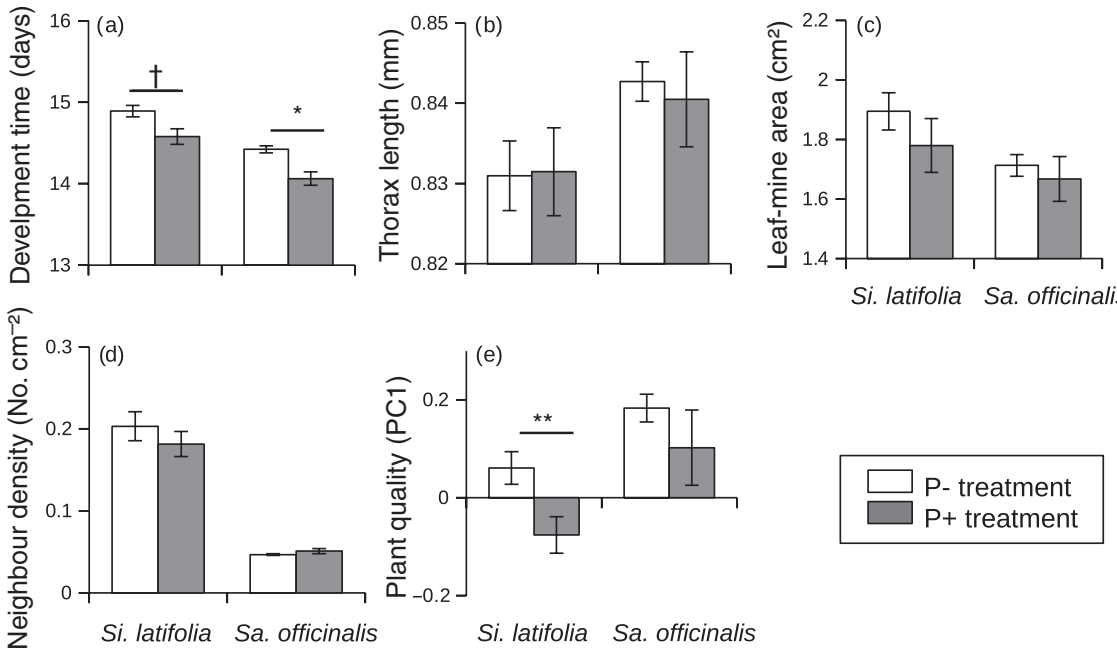

Fig. 1. Effects of exposure to parasitism on five focal traits associated with leafminers: (a) development time, (b) adult size (thorax length), (c) leaf mine area, (d) neighbour density, and (e) plant quality. Trait values of leafminer individuals that survived in the $\mathrm{P}-$ treatment are shown in open bars, and those in the $\mathrm{P}+$ treatment are in grey bars. A significant shift in the trait mean between the exposure treatments indicates that parasitoids disproportionately attack leafminers with respect to that trait. Asterisks indicate significant (or marginal) residual effects of exposure treatment controlling for other focal traits: $\dagger<0.1, *<0.5, * *<0.01$. The error bars represent standard errors.

$\mathrm{P}+$ than in the $\mathrm{P}-$ treatments $\left(F_{1,239}=9.48, P=0.002\right.$, Fig. $\left.1 \mathrm{e}\right)$. On Si. latifolia, plant quality was positively correlated with development time and leafmine area, and negatively correlated with final body size and neighbour density (Table S1).

\section{Effect of plant quality on direct and indirect resistance}

On both Sa. officinalis and Si. Latifolia, in both exposure treatments, larvae consumed more leaf tissue as plant quality decreased $\left(F_{1,45}=31.9, P<0.0001 \& F_{1,54}=27.4, P<0.0001\right.$, Fig. 2a and b, respectively). No effects of exposure treatment or exposure $\times$ plant quality interactions were found on either host species $(F<1.46, P>0.23)$. Larval mortality on Sa. officinalis was higher in $\mathrm{P}+$ than the $\mathrm{P}-$ treatments (d.f. $=74, z=17.2$, $P<0.0001$; Fig. 2c), whereas the overall effect of plant quality was minimal (d.f. $=74, z=1.1, P=0.27$ ). However, plant quality and exposure to parasitism interacted (d.f. $=74, z=-3.48$, $P=0.0005$ ), suggesting that plant quality affects leafminer mortality in a different manner when exposed and protected from parasitism. Post-hoc analyses showed that plant quality did not influence larval mortality in the absence of parasitism (d.f. $=31, z=1.1, P=0.27$; Fig. 2 c dashed line) but was negatively correlated with mortality in the presence of parasitism (d.f. =43, $z=-3.4, P=0.0006$; Fig. 2c, solid line). On Si. latifolia, larval mortality was only associated with exposure treatment (d.f. $=64$, exposure: $z=-9.7, P<0.0001$, plant quality: $z=0.16, P=0.87$, exposure $\times$ plant quality: $z=-0.95$, $P=0.34$; Fig. 2d).

\section{Discussion}

The SGHM hypothesis predicts that prolonged larval stages in insect herbivores will increase mortality from their natural enemies (Moran \& Hamilton, 1980; Clancy \& Price, 1987). I tested the SGHM hypothesis along with four other hypotheses that are germane to parasitism risk in the leafminer, A. flavifrons. On Sa. officinalis, leafminers that survived to adulthood in the presence of parasitism $(\mathrm{P}+)$ had, on average, a shorter development time than those in the absence of parasitism $(\mathrm{P}-)$. A similar trend was also observed on Si. latifolia. Because parasitised leafminers are not likely to survive to an adult stage (i.e. no encapsulation ability observed), the shifts in mean development time indicate that individuals with a longer development time were more likely to be attacked and killed by parasitoids. If the leafminer was capable of encapsulating, however, my estimate of development time difference between exposure treatments would be conservative, because individuals that recovered from parasitism would have delayed larval development owing to the cost of encapsulation (Moret \& Schmid-Hempel, 2000).

Alternatively, the difference in larval development time could indicate behavioural changes in leafminers that are under parasitism risk. Reduced feeding activity to avoid detection may either prolong the larval development time (Laurila et al., 2008) or shorten it by enhancing food assimilation efficiency (Thaler et al., 2012). The latter case would result in overestimation of the SGHM effect while suggesting that leafminer's behavioural change could diminish the opportunity for SGHM to act upon. Although not specifically tested here, chronic risk of parasitism during the entire larval period is not likely to shorten larval period in A. flavifrons, because no difference in overall leaf consumption (Fig. 2a,b) and final body size (Fig. 1b) was observed between survivors from the $\mathrm{P}-$ and $\mathrm{P}+$ treatments.

Finally in Sa. officinalis, the bagging treatment reduced plant quality in $\mathrm{P}+$ compared with the $\mathrm{P}-$ treatment, which could have affected larval development time. However, the analyses used here controlled for such possibility by including plant quality as a covariate when testing for the exposure treatment effect on 
(a)

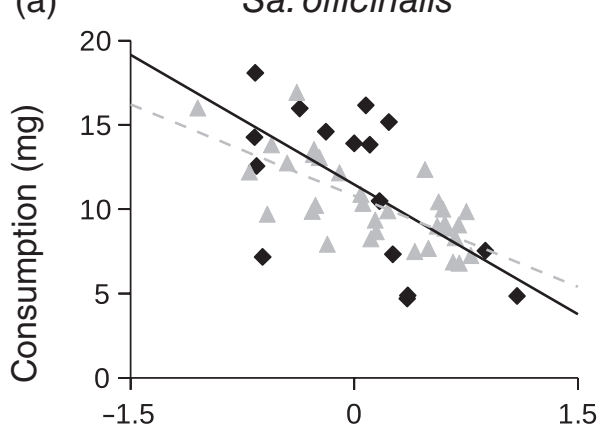

(c)

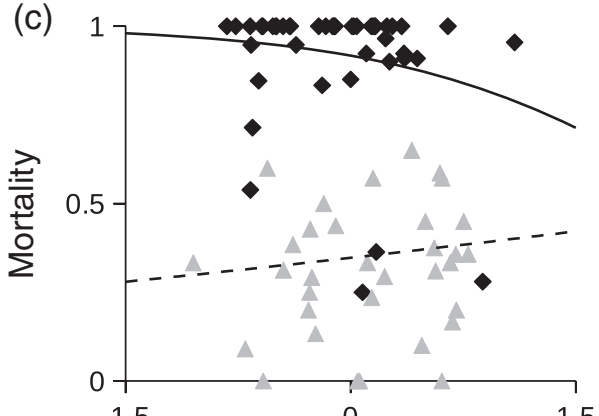

(b)

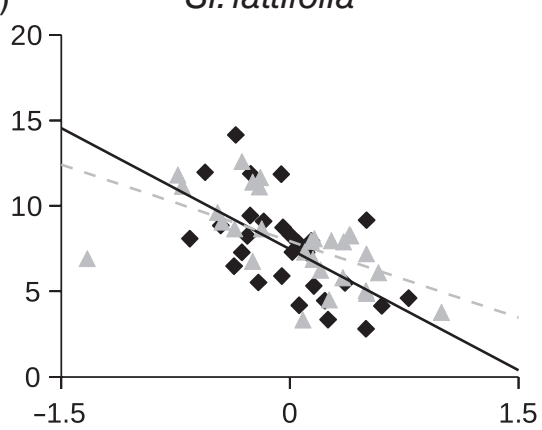

(d)

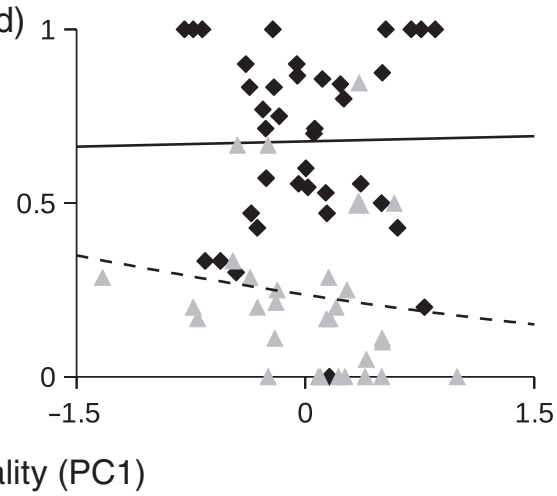

Fig. 2. Relationship between plant quality and leaf tissue consumed (mg) by individual leafminer (a,b) and larval mortality rate (c,d) on Saponaria officinalis (a,c) and on Silene latifolia (b,d). Grey triangles and dashed lines indicate the relationship in the P- treatment, and black diamonds and solid lines indicate that in the P+ treatment. Plant quality was negatively associated with leaf consumption on both host species in both exposure treatments (linear regression models: $F>27.4, P<0.0001$ ). Plant quality was negatively correlated with leafminer mortality only in the P+ treatment on $S a$. officinalis (binomial regression model: $z=-3.4, P=0.0006$ ).

development time. The observed pattern, therefore, supports the SGHM hypothesis that prolonged larval development increases parasitism.

Prior empirical validation of the SGHM hypothesis has been limited overall, and the support for the hypothesis is equivalent for concealed herbivores under parasitoid attacks (53\% of 32 studies supported: reviewed by Williams, 1999). A small number of laboratory studies yielded support for SGHM during critical 'windows of vulnerability', including parasitism of a shoot-galling sawfly by a specialist parasitoid species (Craig et al., 1990), and susceptibility of early instars of the cabbage butterfly to a generalist parasitoid species (Benrey \& Denno, 1997). Within a natural and ecologically relevant context, Clancy and Price (1987) found the opposite of the SGHM prediction: that faster growing leaf-galling sawfly larvae suffered increased field mortality from two specialist parasitoids, which preferentially attacked large and rapidly growing prey. The present study in A. flavifrons demonstrates SGHM acting within the natural context, where leafminers were exposed to a natural assembly of parasitoids across the entire course of larval development, thereby capturing the total effect of parasitoid-induced mortality on larval development time.

While I demonstrated SGHM within host plant species, the pattern disappeared in a contrast between host plant species. Leafminers developed faster on Sa. officinalis (Fig. 1a), yet they were attacked more frequently on this host than on Si. latifolia. Because plants used in this study are likely to represent a limited number of genotypes within each species, contrasts between species requires caution. However, some morphological differences between host plant species may explain the inter-specific variation in parasitism. For example, trichomes that may hinder parasitoids' movements (Carrillo etal., 2012) are absent in Sa. officinalis but present in Si. latifolia, and colour contrasts between the mine and the leaf, which could make leafmines more conspicuous to parasitoids (Salvo \& Valladares, 2004), are greater in Sa. officinalis than in Si. latifolia. Because host plant species could differ in traits influencing attractiveness to parasitoids, studies of the SGHM hypothesis should focus on intra-specific effects (Benrey \& Denno, 1997).

\section{Other factors affecting parasitism}

Some parasitoids are known to use visual cues to locate their prey (Ayabe \& Ueno, 2004), and larger leaf-mines (Cornelissen \& Stiling, 2006) and higher neighbour density (Singer etal., 2012) were hypothesised to increase prey-searching efficiency. Also, these parameters may be positively correlated with induced responses from the host plants, which could further attract enemies owing to induction of volatile organic compounds (VOCs: Dicke \& Minkenberg, 1991; Wei 
\& Kang, 2006). Despite the expectation, I found no evidence of mine-size or larval-density dependent parasitism in A. flavifrons, suggesting that these traits did not influence parasitoid's searching efficiency, at least in the spatial scale employed here. The relationship between feeding intensity and the level of plant VOC induction is unknown in this system, but prolonged larval feeding could attract parasitoids via increased VOC emission, and may provide chemical mechanisms underlying SGHM (Kessler \& Baldwin, 2004; Kaplan et al., 2007).

Several studies suggest that parasitism might be associated with herbivore traits that reflect the availability of nutrients for parasitoid larval development (e.g. prey size and host plant quality; Loader \& Damman, 1991). The present study did not identify any association between prey size and parasitism, which might indicate that the parasitoids of A. flavifrons are generalists that are indifferent to size variation in their prey (Williams, 1999). Alternatively, different parasitoid species may attack leafminers based on different sets of traits, resulting in a net absence of an effect of herbivore body size. There is considerable diversity of parasitoids of A. flavifrons, with multiple species attacking early or late instars of the larvae (e.g. early instars are often eaten by adult parasitoids; at least seven morpho-species of koinobiont and idiobiont species parasitise the later instars). Thus, when the whole parasitoid community is considered, it is unlikely that the 'window of vulnerability' of the leafminer is limited to a single larval size class (as is assumed by Moran \& Hamilton, 1980).

Plant quality directly affected parasitism on Si. latifolia, where larvae that survived in the $\mathrm{P}+$ treatment were associated with a lower plant quality than those in the $\mathrm{P}$ - treatment (Fig. 1e). The observed pattern suggests that parasitoids are actively searching and killing leafminers on plants with higher nutritional quality, supporting the plant quality hypothesis. Surprisingly, plant quality was negatively correlated with the final body size of the leafminer (Table S1), suggesting that searching behaviour based on plant quality does not necessarily result in gaining larger, and potentially higher quality prey. On Sa. officinalis, such a direct effect of plant quality on parasitism was not observed, but plant quality may indirectly affect leafminer's survival via altering their development time. Plant quality was negatively correlated with larval development time, suggesting that leafminers may suffer higher parasitism on low-quality plants owing to SGHM.

\section{Plant nutritional quality and plant resistance}

The SGHM hypothesis was originally motivated by the assumption that low nutritional quality of a plant should provide no benefit to the plant, except in the presence of an interaction with a third trophic level (Moran \& Hamilton, 1980; Price etal., 1980). I conducted a post-hoc analysis to assess whether variation in plant quality generated by nitrogen addition treatments (characterised as the first principal component in a PCA analysis) could function as an indirect resistance against the leafminers on two host plant species.

Individual leafminers consumed more leaf tissues as plant quality decreased on both hosts, and the relationship did not depend on exposure treatments. The results suggest that the low nutritional quality of plants does not function as a direct resistance against the leafminers and that the presence of parasitoids did not influence their feeding behaviour (i.e. no trait-mediated interactions). In contrast, plant quality and parasitoid exposure had an interacting effect on leafminer mortality on $S a$. officinalis. Leafminer mortality increased with a decrease in plant quality only in the presence of parasitoids ( $\mathrm{P}+$ treatment), suggesting that low nutritional quality could function as an indirect plant resistance via tri-trophic interactions. Such indirect resistance was not observed on Si. latifolia, however. This host-species dependent effect on indirect resistance may result from differences in overall parasitoid load, the strength of the SGHM effect, and the differential effect of plant quality on leafminer development time. In fact, plant quality was negatively correlated with larval development time on Sa. officinalis, but no correlation was found on Si. latifolia. An interpretation of the observed differences between host plant species requires some caution because plants used in this experiment represent only a small genotypic variation (if any) within a species, and different plant genotypes are likely to differ in mode of resistance against herbivores (Gols et al., 2008). Further demonstrations of SGHM in relation to plant nutritional quality using plant genotypes that vary in primary, as well as secondary metabolites, could provide new insights into the evolution of low plant quality as mechanisms of direct and indirect plant defences (Wise et al., 2006).

\section{Acknowledgement}

I thank Beverly Rathcke, Tim Connallon, and Rayko Halitschke for helpful discussions and suggestions on earlier drafts of this study, and Caroline Cheng for assistance in the field. Leaf chemical analyses were performed in the lab of Mark Hunter and Knute Nadelhoffer, with assistance from Jim Le Moine and Sarah Breed. This study was funded with Rackham Graduate Student Research Grants and Mathaei Botanical Gardens Research Grants at the University of Michigan.

\section{Supporting Information}

Additional Supporting Information may be found in the online version of this article under the DOI reference:

10.1111/een. 12177

Table S1. Results of LMM analyses testing for (1) SGHM, (2) prey size, (3) mine appearance, (4) density-dependent parasitism, and (5) plant quality hypotheses on Sa. officinalis and Si. latifolia.

\section{References}

Auerbach, M. \& Alberts, J. (1992) Occurrence and performance of the aspen blotch miner, Phyllonorycter salicifoliella, on three host-tree species. Oecologia, 89, 1-9.

Ayabe, Y. \& Ueno, T. (2004) Directed search pattern of a leafminer parasitoid among mines of host larvae. Annals of the Entomological Society of America, 97, 586-591.

Benrey, B. \& Denno, R. (1997) The slow-growth-high-mortality hypothesis: a test using the cabbage butterfly. Ecology, 78, 987-999. 
Carrillo, J., Wang, Y., Ding, J., Klootwyk, K. \& Siemann, E. (2012) Decreased indirect defense in the invasive tree, Triadica sebifera. Plant Ecology, 213, 945-954.

Clancy, K. \& Price, P. (1987) Rapid herbivore growth enhances enemy attack - sublethal plant defenses remain a paradox. Ecology, $\mathbf{6 8}$, $733-737$.

Cornelissen, T. \& Stiling, P. (2006) Does low nutritional quality act as a plant defence? An experimental test of the slow-growth, high-mortality hypothesis. Ecological Entomology, 31, 32-40.

Craig, T., Itami, J. \& Price, P. (1990) The window of vulnerability of a shoot-galling sawfly to attack by a parasitoid. Ecology, 71, $1471-1482$.

Dicke, M. \& Minkenberg, O.P.J.M. (1991) Role of volatile infochemicals in foraging behavior of the leafminer parasitoid Dacnusa sibirica; (Diptera: Agromyzidae). Journal of Insect Behavior, 4, 489-500.

Feeny, P. (1976) Plant apparency and chemical defense. Recent Advances in Phytochemistry, 10, 1-40.

Goławska, S., Łukasik, I. \& Leszczyński, B. (2008) Effect of alfalfa saponins and flavonoids on pea aphid. Entomologia Experimentalis et Applicata, 128, 147-153.

Gols, R., Wagenaar, R., Bukovinszky, T., van Dam, N.M., Dicke, M., Bullock, J.M. et al. (2008) Genetic variation in defense chemistry in wild cabbages affects herbivores and their endoparasitoids. Ecology, 89, 1616-1626.

Hunter, M.D. (2003) Effects of plant quality on the population ecology of parasitoids. Agricultural and Forest Entomology, 5, 1-8.

Hunter, M. \& Price, P. (1992) Playing chutes and ladders - heterogeneity and the relative roles of bottom-up and top-down forces in natural communities. Ecology, 73, 724-732.

Jervis, M. \& Kidd, N. (1986) Host-feeding strategies in hymenopteran parasitoids. Biological Reviews of the Cambridge Philosophical Society, 61, 395-434.

Jia, Z.H., Koike, K. \& Nikaido, T. (1998) Major triterpenoid saponins from Saponaria officinalis. Journal of Natural Products, 61, $1368-1373$.

Jia, Z., Koike, K., Sahu, N.P. \& Nikaido, T. (2002) Triterpenoid saponins from Caryophyllaceae family. Studies in Natural Products Chemistry, Bioactive Natural Products (ed. by Atta-ur-Rahman), pp. 3-61. Elsevier Science Publishers, Amsterdam, The Netherlands.

Johnson, M. \& Gould, F. (1992) Interaction of genetically engineered host plant-resistance and natural enemies of Heliothis virescens (lepidoptera, noctuidae) in tobacco. Environmental Entomology, 21, 586-597.

Kaplan, I., Lynch, M., Dively, G. \& Denno, R. (2007) Leafhopper-induced plant resistance enhances predation risk in a phytophagous beetle. Oecologia, 152, 665-675.

Kessler, A. \& Baldwin, I.T. (2004) Herbivore-induced plant vaccination. Part I. The orchestration of plant defenses in nature and their fitness consequences in the wild tobacco Nicotiana attenuata. Plant Journal, 38, 639-649.

Kraaijeveld, A.R. \& Godfray, H.C.J. (2003) Potential life-history costs of parasitoid avoidance in Drosophila melanogaster. Evolutionary Ecology Research, 5, 1251-1261.

Laurila, A., Lindgrenm, B. \& Laugen, A.T. (2008) Antipredator defenses along a latitudinal gradient in Rana temporaria. Ecology, 89, 1399-1413.

Loader, C. \& Damman, H. (1991) Nitrogen-content of food plants and vulnerability of Pieris rapae to natural enemies. Ecology, 72, $1586-1590$.

Medina, R., Barbosa, P. \& Waddell, K. (2005) Parasitism levels in Orgyia leucostigma feeding on two tree species: implications for the slow-growth-high-mortality hypothesis. Entomologia Experimentalis et Applicata, 115, 193-197.

Moran, N. \& Hamilton, W.D. (1980) Low nutritive quality as defense against herbivores. Journal of Theoretical Biology, 86, 247-254.

Moret, Y. \& Schmid-Hempel, P. (2000) Survival for immunity: the price of immune system activation for bumblebee workers. Science, $\mathbf{2 9 0}$, $1166-1168$.

Otway, S.J., Hector, A. \& Lawton, J.H. (2005) Resource dilution effects on specialist insect herbivores in a grassland biodiversity experiment. Journal of Animal Ecology, 74, 234-240.

Price, P., Bouton, C., Gross, P., McPheron, B., Thompson, J. \& Weis, A. (1980) Interactions among three trophic levels: influence of plants on interactions between insect herbivores and natural enemies. Anпиаl Review of Ecology and Systematics, 11, 41-65.

de Roode, J.C., Pedersen, A.B., Hunter, M.D. \& Altizer, S. (2008) Host plant species affects virulence in monarch butterfly parasites. Journal of Animal Ecology, 77, 120-126.

Salvo, A. \& Valladares, G. (2004) Looks are important: parasitic assemblages of agromyzid leafminers (Diptera) in relation to mine shape and contrast. Journal of Animal Ecology, 73, 494-505.

Scheffer, S. (1995) Genetic and Ecological Influences of the Evolution of Host Range in a Leafmining Fly. State University of New York at Stony Brook, Stony Brook, New York.

Singer, M.S., Farkas, T.E., Skorik, C.M. \& Mooney, K.A. (2012) Tritrophic interactions at a community level: effects of host plant species quality on bird predation of caterpillars. American Naturalist, 179, 363-374.

Slansky, F. (1986) Nutritional ecology of endoparasitic insects and their hosts: an overview. Journal of Insect Physiology, 32, 255-261.

Spencer, K. (1990) Host Specialization in the World Agromyzidae (Diptera). Kluwer Academic Publishers, Boston, Massachusetts.

Stiling, P., Rossi, A.M., Hungate, B., Dijkstra, P., Hinkle, C.R., Knott, W.M. et al. (1999) Decreased leaf-miner abundance in elevated CO2: reduced leaf quality and increased parasitoid attack. Ecological Applications, 9, 240-244.

Thaler, J.S., McArt, S.H. \& Kaplan, I. (2012) Compensatory mechanisms for ameliorating the fundamental trade-off between predator avoidance and foraging. Proceedings of the National Academy of Sciences, 109, 12075-12080.

Walker, M., Hartley, S. \& Jones, T. (2008) The relative importance of resources and natural enemies in determining herbivore abundance: thistles, tephritids and parasitoids. Journal of Animal Ecology, 77, $1063-1071$.

Wang, X.G. \& Messing, R.H. (2004) Fitness consequences of body-size-dependent host species selection in a generalist ectoparasitoid. Behavioral Ecology and Sociobiology, 56, 513-522.

Wei, J.N. \& Kang, L. (2006) Electrophysiological and behavioral responses of a parasitic wasp to plant volatiles induced by two leaf miner species. Chemical Senses, 31, 467-477.

Williams, I. (1999) Slow-growth, high-mortality-a general hypothesis, or is it? Ecological Entomology, 24, 490-495.

Wise, M.J., Fox, R.J. \& Abrahamson, W.G. (2006) Disarming the paradox of sublethal plant defense against insects: Trirhabda virgata larval development time and leaf tissue loss on Solidago altissima. Entomologia Experimentalis et Applicata, 120, 77-87.

Wolfe, L.M. (2002) Why alien invaders succeed: support for the escape-from-enemy hypothesis. The American Naturalist, 160, $705-711$.

Accepted 19 November 2014

First published online 12 February 2015 